\title{
Advanced glycation end products and the absence of premature atherosclerosis in glycogen storage disease Ia
}

\author{
N. C. den Hollander • D. J. Mulder • R. Graaff • \\ S. R. Thorpe • J. W. Baynes • G. P. A. Smit • A. J. Smit
}

Received: 13 November 2006/Submitted in revised form: 8 March 2007 / Accepted: 19 April 2007 / Published online: 14 June 2007

(C) SSIEM and Springer 2007

Summary Introduction: Despite their unfavourable
cardiovascular risk profile, patients with glycogen
storage disease type Ia (GSD Ia) do not develop
premature atherosclerosis. We hypothesized that this
paradox might be related to a decreased formation of
advanced glycation end products (AGEs) resulting
from lifetime low plasma glucose levels and decreased
oxidative stress. Methods: In 8 GSD Ia patients (age

Communicating editor: René Santer

Competing interests: R. Graaff and A.J. Smit are founders of DiagnOptics B.V., The Netherlands, manufacturer of the AGE- Reader

References to electronic databases: Glycogen storage disease type Ia (GSD Ia): OMIM +232200. Glucose-6-phosphatase (G6Pase) EC 3.1.3.9.

N. C. den Hollander • D. J. Mulder · A. J. Smit $(\bowtie)$

Department of Internal Medicine,

University Medical Center Groningen, Hanzeplein 1,

PO Box 30.001, 9700 RB Groningen, The Netherlands

e-mail: a.j.smit@int.umcg.nl

\section{R. Graaff}

Department of Biomedical Engineering,

University Medical Center Groningen,

Groningen, The Netherlands

S. R. Thorpe $\cdot$ J. W. Baynes

Department of Chemistry and Biochemistry,

University of South Carolina, Columbia,

South Carolina, USA

G. P. A. Smit

Department of Pediatrics, University Medical Center

Groningen, Groningen, The Netherlands
20-34 years) and 30 matched controls we measured carotid intima-media thickness (IMT), skin autofluorescence (AF; a non-invasive index for AGEs), and specific AGEs (pentosidine, $N$-(carboxymethyl)lysine (CML), $N$-(carboxyethyl)lysine (CEL)) and collagen linked fluorescence (CLF, measured at excitation/ emission wavelength combinations of $328 / 378$ and 370/440 nm) in skin samples. Results: Carotid IMT was significantly lower in GSD Ia patients. Skin AF did not differ between patients and controls. The skin samples showed higher CEL levels in the patient group ( $p=0.008$ ), but similar levels of pentosidine, CML, and CLF. In the total group, skin AF correlated with CML $(r=0.39, p=0.031)$, CLF $328 / 378 \mathrm{~nm}(r=0.53 ; p=0.002)$ and CLF $370 / 440 \mathrm{~nm}(r=0.60 ; p=0.001)$. In the control group, AF also correlated with the maximum carotid IMT ( $r=0.6 ; p=0.004)$. Conclusion: Although our data confirm that GSD Ia patients present with a reduced burden of atherosclerosis, this phenomenon cannot be explained by differences in AGE accumulation as measured in the skin.

$\begin{array}{ll}\text { Abbreviations } \\ \text { AF } & \text { autofluorescence } \\ \text { AGEs } & \text { advanced glycation end products } \\ \text { AU } & \text { arbitrary units } \\ \text { CB } & \text { carotid bulb } \\ \text { CCA } & \text { common carotid arteries } \\ \text { CEL } & N \text {-(carboxyethyl)lysine } \\ \text { CLF } & \text { collagen linked fluorescence } \\ \text { CML } & N \text {-(carboxymethyl)lysine } \\ \text { G6Pase } & \text { glucose-6-phosphatase } \\ \text { GSD Ia } & \text { glycogen storage disease type Ia }\end{array}$


HbA1c glycated haemoglobin A1c

HDL high-density lipoprotein

ICA internal carotid artery

IMT intima-media thickness

RAGE receptor for advanced glycation end products

\section{Introduction}

Glycogen storage disease type Ia (GSD Ia, von Gierke disease; OMIM +232200) is an inborn error of metabolism, caused by deficiency of microsomal glucose-6phosphatase (G6Pase; EC 3.1.3.9) in liver and kidney. G6Pase is a key enzyme in homeostatic regulation of blood glucose concentration by catalysing the terminal step in both glycogenolysis and gluconeogenesis.

Although the main feature of this disease is severe fasting hypoglycaemia, several other metabolic changes complicate the disease. GSD Ia patients have marked hyperlipidaemia, characterized by a high total cholesterol with a low level of high-density lipoprotein (HDL) and, most strikingly, high triglyceride levels, a result of both increased synthesis and, possibly, decreased plasma lipid clearance. Another long-term complication is renal failure. After a silent period of hyperfiltration, patients develop microalbuminuria and eventually frank proteinuria during the first three decades of life. Dyslipidaemia and microalbuminuria are known to be strong risk factors for atherosclerosis and cardiac disease (Verhave et al 2005; Yudkin et al 1988). As patients are surviving longer, the concern has risen that GSD Ia patients may be at high risk for atherosclerosis as well (Levy et al 1988). However, in contrast to other diseases associated with exposure to cardiovascular risk factors at an early age, such as familial hypercholesterolaemia (Wiegman et al 2004), it has been shown that GSD Ia patients do not show any signs of endothelial dysfunction or premature atherosclerosis (Lee 1994; Talente et al 1994; Ubels et al 2002).

Recently, the accumulation of advanced glycation end products (AGEs) on tissue proteins has been implicated as a contributing factor in atherosclerosis (Forbes et al 2004; Kiuchi et al 2001; Nakamura et al 1993; Ritthaler et al 1995). AGEs are a heterogeneous group of compounds formed by the Maillard reaction, or the non-enzymatic browning reaction that modifies proteins with carbohydrate-and lipid-derived intermediates (Thorpe and Baynes 2003). AGE formation is increased in hyperglycaemia and under the influence of oxidative stress (Elgawish et al 1996; Thorpe and Baynes 2003; Wells-Knecht et al 1995, 1997; Wolff and
Dean 1987). AGE formation alters the characteristics of both short- and long-lived (e.g. collagen) proteins. AGEs accumulate in extracellular matrix proteins but also in atherosclerotic plaques (Nakamura et al 1993; Schleicher et al 1997) and the expression of receptors for AGEs (RAGE) is increased in atherosclerosis (Ritthaler et al 1995). Interaction of AGE-modified proteins with AGE receptors may stimulate cytokine and growth factor production that sustains the development of the atherosclerotic plaques.

We therefore postulated that as increased AGE formation leads to atherosclerosis, decreased AGE formation might protect against this disease. We hypothesized that GSD Ia patients have lower AGE levels based on two observations. First, GSD Ia patients may be more frequently hypoglycaemic (Hershkovitz et al 2001; Rake et al 2002). Second and most importantly, GSD Ia patients have significantly lower levels of oxidative stress (Wittenstein et al 2002). The finding of low AGE levels in women with polycystic ovary syndrome, a common complication in GSD Ia (Rake et al 2002) that also increases the risk for atherosclerosis, also contributes to this hypothesis (Diamanti-Kandarakis et al 2005).

In the current study, we assessed AGEs in tissue both by quantification of specific AGEs and measurement of collagen linked fluorescence (CLF) in skin biopsies, and by measuring skin autofluorescence (AF), using a recently developed and validated method (Mulder et al 2006). We aimed to investigate whether patients with GSD Ia present with lower levels of AGE accumulation compared with age- and sex-matched healthy controls.

\section{Methods}

Subjects

A total of 38 adults were enrolled in this study, 8 GSD

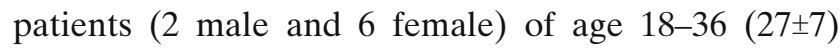
years and 30 healthy control subjects matched for age and sex ( 8 male and 22 female). All GSD patients aged $\geq 18$ years, who attended our outpatient clinic or were admitted during the study period, were approached for participation and age-matched controls were approached after the selection of individual patients. All participants were assessed with the procedures described below during the same study period. Relevant information on medical history and smoking habits was obtained from both patients and control 
subjects. The study was approved by the local Medical Ethical Committee and written informed consent was obtained from all participants.

\section{Skin autofluorescence (AF)}

Skin AF was assessed on the ventral site of the lower arm with a prototype of the current AGE-Reader (DiagnOptics BV, Groningen, The Netherlands) as described elsewhere (Meerwaldt et al 2004, 2005b; Mulder et al 2006). In short, the AGE-Reader consists of a tabletop box containing a black light excitation light source (peak wavelength $\sim 360 \mathrm{~nm}$ ). Light emitted from the skin is measured with an integrated spectrometer. Measurement is fully automated and takes approximately $30 \mathrm{~s}$ to perform, giving an average value over 50 individual scans. Skin AF is calculated by dividing the mean value of the emitted light intensity per $\mathrm{nm}$ between 420 and $600 \mathrm{~nm}$ by the mean value of the excitation light intensity per nm between $300420 \mathrm{~nm}$, expressed as arbitrary units (AU). The intra-individual Altman error percentage is $5.0 \%$ on a single day and $5.9 \%$ for seasonal changes (Meerwaldt et al 2004).

\section{Carotid intima-media thickness (IMT) measurement}

IMT of the carotid arteries was measured by ultrasonography in the supine position. High-resolution B-mode ultrasound imaging (ACUSON 128 XP; Acuson Corp, Mountain View, CA, USA) with a 7.0 $\mathrm{MHz}$ linear array transducer was used to measure intima-media wall thickness, as described previously in greater detail (Terpstra et al 2004). In short, the right and left common carotid arterial wall segments were imaged from a fixed lateral transducer angle at the far wall of the distal $1 \mathrm{~cm}$ segments of both common carotid arteries (CCA), proximal to the carotid bulb. The far wall of the carotid bulb (CB), and of the most proximal part of the internal carotid artery (ICA) were also scanned bilaterally. The scans were recorded on S-VHS tape and analysed offline by an independent image analyst unaware of subject characteristics. B-mode image analyses were digitized with a frame grabber (DT286 1; Data Translation, Marlboro, MA, USA). The image analysis software was developed using an algorithm developed by Selzer and colleagues (Selzer et al 1994) The average and maximum carotid IMT over the six segments (CCA, CB and ICA, bilaterally far wall) of both carotid arteries were calculated. At a mean IMT of $0.80 \mathrm{~mm}$, intersonographer variability amounted to $0.05 \mathrm{~mm}$, with an image analyst variability $<0.03 \mathrm{~mm}$, corresponding to a total variation coefficient between $6.3 \%$ and $7.3 \%$.

Blood and urine collection

Venous blood and a portion of urine were taken nonfasting to measure glycated haemoglobin A1c (HbA1c), lipid spectrum, creatinine and urine albumin, using routine laboratory methods.

Skin samples and measurement of AGEs

A $4 \mathrm{~mm}$ diameter full-thickness skin biopsy was taken under $2 \%$ lidocaine-adrenaline anaesthesia from the volar (frontal) side of the lower arm. The sample was snap-frozen in liquid nitrogen and stored at $-80^{\circ} \mathrm{C}$. After thawing, insoluble collagen was extracted as previously described (Fu et al 1994; Meerwaldt et al 2004); the samples were reduced with sodium borohydride and solubilized using pepsin. Then collagen linked fluorescence (CLF) was measured using wavelengths of 328/378 nm and 370/440 nm (excitation/ emission). Both CLF and pentosidine were normalized to the hydroxyproline content of the sample measured according to Stegemann and Stalder (1967). Pentosidine was measured by high-performance liquid chromatography as described by Dyer and colleagues (1993). $N$-(Carboxymethyl)lysine (CML) and $N$ (carboxyethyl)lysine (CEL) analysis was performed by gas chromatography-mass spectrometry as described by Dunn and colleagues (Ahmed et al 1997). CML and CEL were normalized to the lysine content of the sample.

\section{Statistical analysis}

Owing to the low prevalence of the disease, we could not include more than 8 adult patients with GSD Ia for this study. We determined that with this number of patients and an expected standard deviation of 0.25 , a sample size of 30 controls would be needed to give the study $80 \%$ power at $\alpha=0.05$ to detect at least a difference of $20 \%$ between both groups.

Because of the small group measured, data are given as medians (interquartile range). Mann-Whitney test was performed to compare groups and Spearman's rho test to calculate correlations. All analyses were performed with SPSS version 13.0 for Windows (SPSS, Chicago, IL, USA). A value of $p<0.05$ was considered statistically significant. 
Table 1 Characteristics of patients and controls

\begin{tabular}{llll}
\hline & Controls $(n=29)$ & Patients $(n=8)$ & $p$-Value \\
\hline Age $($ years $)$ & $25(21-32)$ & $27(20-34)$ & NS \\
Length $(\mathrm{m})$ & $1.78(1.69-1.85)$ & $1.66(1.61-1.68)$ & 0.019 \\
Sex $($ male/female) & $8 / 21$ & $2 / 6$ & $\mathrm{NS}$ \\
Body mass index $\left(\mathrm{kg} / \mathrm{m}^{2}\right)$ & $23(21-26)$ & $24(22-28)$ & $\mathrm{NS}$ \\
Smoking $(n)$ & 1 & $4.8(4.6-5.3)$ & $\mathrm{NS}$ \\
HbA1c $(\%)$ & $4.9(4.6-5.0)$ & $4.05(3.07-7.10)$ & $\mathrm{NS}$ \\
Triglycerides $(\mathrm{mmol} / \mathrm{L})$ & $1.19(0.88-1.63)$ & $5.88(5.22-7.72)$ & $<0.001$ \\
Cholesterol $(\mathrm{mmol} / \mathrm{L})$ & $4.90(4.15-5.35)$ & $1.14(1.00-1.25)$ & 0.012 \\
HDL-cholesterol $(\mathrm{mmol} / \mathrm{L})$ & $1.44(1.32-1.76)$ & $3.07(1.61-1.68)$ & 0.008 \\
LDL-cholesterol $(\mathrm{mmol} / \mathrm{L})$ & $2.53(2.10-3.04)$ & $143(123-153)$ \\
Creatinine $(\mu \mathrm{mol} / \mathrm{L})$ & $81(75-92)$ & $1.2(0.6-6.9)$ & $\mathrm{NS}$ \\
Creatinine clearance $(\mathrm{ml} / \mathrm{min})^{\mathrm{a}}$ & $125(112-142)$ & $0.53(0.48-0.59)$ & 0.002 \\
Albumin/creatinine ratio & $0.8(0.5-1.2)$ & $0.68(0.60-0.74)$ & $\mathrm{NS}$ \\
Average carotid IMT $(\mathrm{mm})$ & $0.60(0.58-0.62)^{\mathrm{b}}$ & $0.77(0.74-0.81)^{\mathrm{b}}$ & 0.008 \\
Maximum carotid IMT $(\mathrm{mm})$ & & 0.004 \\
\hline
\end{tabular}

Data are given as median and interquartile range or as absolute numbers. Differences between groups are calculated with the MannWhitney $U$-test or chi-squared test where appropriate.

IMT, intima media thickness; HDL, high-density lipoprotein; LDL, low-density lipoprotein; HbA1c, glycated haemoglobin A1c, NS, not significant.

${ }^{a}$ Creatinine clearance as calculated by the Cockcroft-Gault formula.

${ }^{\mathrm{b}} n=27$.

\section{Results}

One control subject was excluded because microalbuminuria appeared to be present. The results of the skin biopsy measurements from two male subjects were excluded because the amount of material was not sufficient to provide reliable data, based on hydroxyproline. No sex-based differences were found.

All patients used a xanthine-oxidase inhibitor and 7 out of 8 patients also used an angiotensin-converting enzyme inhibitor. The observed hyperlipidaemia was not treated in any of the patients. Other characteristics of both patient and control groups are listed in Table 1, which shows that the GSD patients enrolled in this study had similar levels of HbA1c. The patients were markedly dyslipidaemic with higher triglycerides, total cholesterol and lower HDL cholesterol compared to controls. The maximum carotid IMT and the average carotid IMT in patients were significantly lower than in the control group.

\section{Skin AGEs measured in skin samples}

Table 2 demonstrates that skin samples of patient versus control subjects showed comparable amounts of the AGEs pentosidine and CML. CEL levels were significantly higher in the patient group (see Fig. 1). No differences were found between patients and

Table 2 Characteristics of patients and controls

\begin{tabular}{|c|c|c|c|}
\hline & Controls $(n=29)$ & Patients $(n=8)$ & $p$-Value \\
\hline Autofluorescence $\left(\times 10^{-2} \mathrm{AU}\right)$ & $1.55(1.30-1.76)$ & $1.67(1.57-1.76)$ & NS \\
\hline Pentosidine ( $\mu \mathrm{g} / \mathrm{g}$ hydroxyproline) & $23.8(18.0-30.6)^{\mathrm{a}}$ & $19.1(16.7-28.4)^{\mathrm{b}}$ & NS \\
\hline $\mathrm{CML}(\mathrm{mmol} / \mathrm{mol}$ lysine $)$ & $1.01(0.81-1.47)^{\mathrm{a}}$ & $1.14(0.78-1.59)^{\mathrm{b}}$ & NS \\
\hline CEL (mmol/mol lysine) & $0.05(0.05-0.07)^{\mathrm{a}}$ & $0.09(0.05-0.13)^{\mathrm{b}}$ & 0.008 \\
\hline 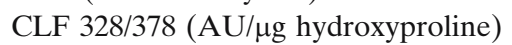 & $324(239-388)$ & $318(286-374)^{\mathrm{b}}$ & NS \\
\hline 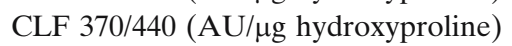 & $203(163-259)$ & $197(179-324)^{\mathrm{b}}$ & NS \\
\hline
\end{tabular}

Data are given as median and interquartile range and differences between groups are calculated with the Mann-Whitney $U$-test. CML, $N$-(carboxymethyl)lysine; CEL, $N$-(carboxyethyl)lysine; CLF, collagen linked fluorescence; AU, arbitrary units; NS, not significant.

${ }^{a} n=27$.

${ }^{\mathrm{b}} n=7$. 


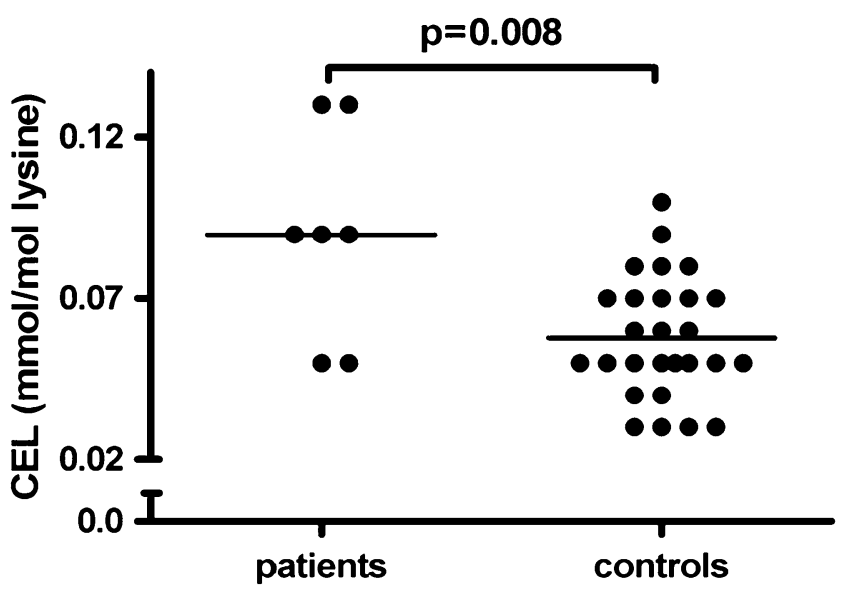

Fig. 1 The amount of $N$-(carboxyethyl)lysine (CEL) in skin samples of the patients and controls; $p$-value was calculated with the Mann-Whitney $U$-test

controls in CLF 328/378 or CLF 370/440. Skin AF did not show any differences between patients and controls (see Fig. 2).

Correlations between indices for AGE accumulation and IMT

In patients and controls taken together, skin $\mathrm{AF}$ correlated with CLF 370/440 $(r=0.62 ; p<0.001)$ and also with CML $(r=0.39 ; p=0.031)$ and CLF 328/278 $(r=0.53$; $p=0.002$ ) (Table 3). Skin AF did not correlate with pentosidine or CEL. All AGEs measured from skin biopsies correlated significantly with each other, apart from CEL which did not correlate with pentosidine.

In the control group, skin AF correlated with maximum carotid IMT ( $r=0.60 ; p=0.004$, see Fig. 3$)$, but not with mean carotid IMT $(r=0.33 ; p=0.139)$. Also, in patients and controls together there was no correlation between carotid IMT and skin AF.

\section{Discussion}

In the current study we confirm the previous observation (Ubels et al 2002) that patients with GSD Ia have a thinner IMT than healthy controls. This supports the finding that these patients, despite severe hyperlipidaemia and microalbuminuria, do not develop premature atherosclerosis.

Since GSD Ia patients have lower levels of wholebody oxidative stress (Wittenstein et al 2002) and a strong tendency to develop hypoglycaemia (Rake et al 2002), we hypothesized that decreased formation of AGEs might protect them from developing premature atherosclerosis. The data from this study do not support this hypothesis. GSD Ia patients did not have lower skin levels of AGEs, as measured either noninvasively using skin AF or ex vivo in skin samples.

Our data confirm the clinical usefulness of skin AF as a tool for measuring AGE accumulation in the skin, since skin AF correlated strongly with the important representative of the AGEs, CML, and with CLF in skin biopsies. Additionally, skin AF correlated with maximum carotid IMT in healthy controls, suggesting it may be non-invasive index to assess cardiovascular risk in apparently healthy subjects. The measurement of skin AF with the AGE-reader is a novel procedure and it may prove to be a useful tool, since the measurement of AGEs in skin samples has several obvious limitations, including its invasive nature. The AGEreader has been validated in diabetic patients, in haemodialysis patients and in healthy controls (Meerwaldt et al 2004, 2005a). In these validation studies, AF correlated with the AGEs pentosidine, CML and CEL. Additionally, skin AF was related to clinical progression of atherosclerotic disease, since it predicted the incidence of future cardiovascular morbidity and mortality in both patient groups (Meerwaldt et al 2005a, 2007). In the current study, we confirm that skin AF correlates with CML and CLF. However it did not correlate with CEL and pentosidine. A potential explanation might be a combination of the small sample size and the narrow and lower range of CEL and pentosidine in the current young group with a small age range compared to previous studies.

Skin biopsies are a generally well-accepted standard for quantifying AGE accumulation in humans. Through this method, we found that only CEL is different in GSD Ia patients. Pentosidine, the AGE that is exclusively derived from carbohydrates, does

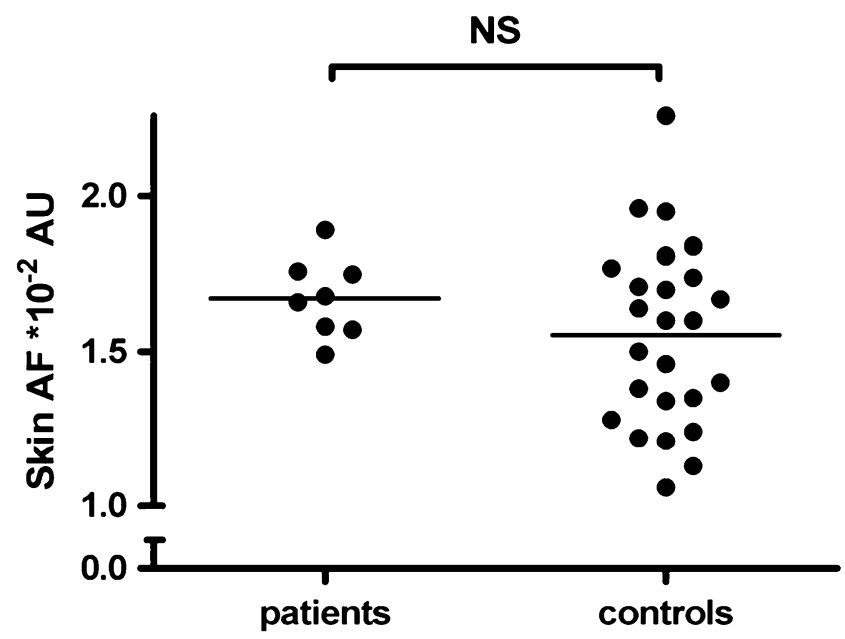

Fig. 2 Skin autofluorescence (AF) in patients and controls; $p$ value was calculated with the Mann-Whitney $U$-test. AU indicates arbitrary units 
Table 3 Correlations between skin autofluorescence (AF) and AGEs in skin biopsies in patients and controls

\begin{tabular}{llllll}
\hline & Skin AF & CLF 370/440 & CLF 328/378 & Pentosidine & CML \\
\hline CLF 370/440 & $0.62(<0.001)$ & & & & \\
CLF 328/378 & $0.53(0.002)$ & $0.96(<0.001)$ & & & \\
Pentosidine & $0.26(\mathrm{NS})$ & $0.61(<0.001)$ & $0.71(<0.001)$ & & \\
CML & $0.39(0.031)$ & $0.59(<0.001)$ & $0.64(<0.001)$ & $0.53(0.001)$ & \\
CEL & $0.28(\mathrm{NS})$ & $0.51(0.002)$ & $0.52(0.001)$ & $0.3(\mathrm{NS})$ & $0.71(<0.001)$ \\
\hline
\end{tabular}

not differ between patient and control groups. CML and CEL are both carbohydrate- and lipid-derived. The hyperlipidaemia in GSD Ia patients might therefore explain the elevated CEL levels in skin biopsies. Our data also show a correlation between triglyceride levels and CEL (data not shown). However, the normal CML levels cannot be explained by this theory.

AGE-formation is increased in hyperglycaemia and during oxidative stress. As expected from current treatment strategies, HbA1c levels in the GSD patients are normal and comparable to those of the controls, which makes important differences in glycaemic stress less likely.

In diabetes, elevated AGE levels in skin collagen are strongly associated with long-term macro- and microvascular complications (Monnier et al 1986). Normal (or even partially elevated) AGEs in GSD Ia patients are not of any help to explain the absence of atherosclerosis. Wittenstein and colleagues compared plasma antioxidants in paediatric patients with GSD Ia, diabetes mellitus and hypercholesterolaemia to clarify the absence of atherosclerosis (Wittenstein et al 2002). GSD Ia patients in their study had increased antioxidative defence in plasma, as measured by the total radical-trapping antioxidant parameter. However, these might have been strongly influenced

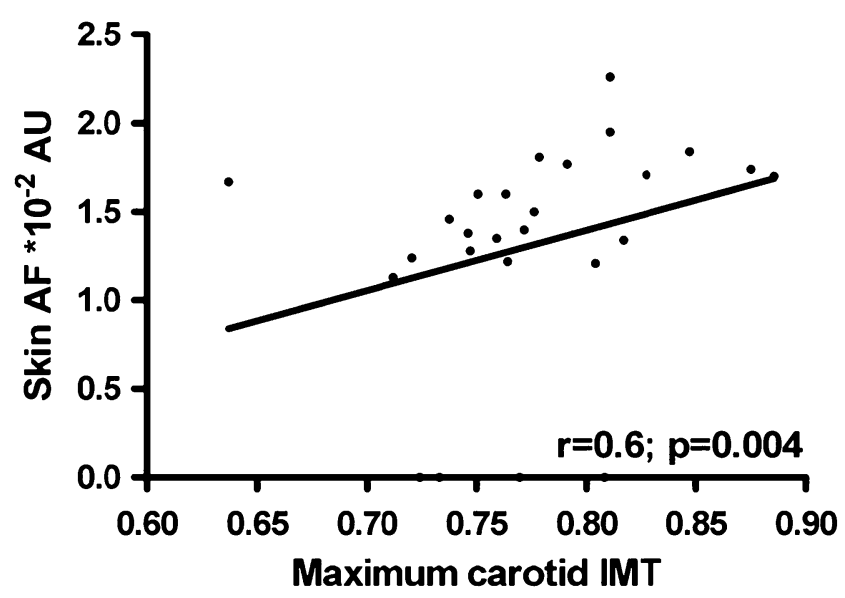

Fig. 3 The correlation between skin autofluorescence (AF) and maximum carotid intima-media thickness (IMT) in control subjects only by elevated uric acid levels in GSD Ia, which may be protective against atherosclerosis (Nieto et al 2000). In our patient group, all patients used the xanthineoxidase inhibitor allopurinol to reduce uric acid levels in order to prevent gout development, which by itself also may reduce oxidative stress and has been shown to improve endothelial dysfunction (Butler et al 2000; Cardillo et al 1997). These factors may have blurred the results from our study.

We found little difference in AGE levels between GSD Ia patients and controls. The extent of renal disease in GSD Ia also has to be taken into account for understanding this result, since AGE accumulation is dependent on renal function (Miyata et al 1998). The contribution of the risk factor microalbuminuria is attenuated, because almost all patients used an ACEinhibitor. In our study, the albumin/creatinine ratio in patients did not differ from the ratio in the controls. The GSD Ia patients in this study did have a significantly lower creatinine, although the creatinine clearance calculated by the Cockcroft-Gault formula did not show any differences from controls. Kidney disease in GSD Ia is known to start with a period of silent hyperfiltration (Baker et al 1989; Chen et al 1988; Mundy and Lee 2002). During this period of hyperfiltration, serum AGE levels may be lower, but whether this may have influenced skin AGEs is uncertain. AGEs linked to skin collagen are generally assumed to be an adequate reflection of overall AGE status because of the long lifespan of this protein. Measurement of AGEs in kidney and liver might reveal aberrant levels of AGEs, because glucose 6phosphate and glycogen are known to accumulate in the liver. Glucose 6-phosphate has a higher potential of forming AGEs than glucose, so AGEs in kidney and liver tissue might be increased in GSD Ia patients. Measurement of AGEs in liver, kidney and urine might help to understand the role of AGEs in GSD Ia patients. In support of this, high levels of detoxification products of the Maillard reaction have been found in a patient with glycogen accumulation in renal tubular cells comparable to GSD Ia (Berry et al 2005).

In conclusion, our study confirms that patients with GSD Ia have a slower progression of atherosclerosis 
compared with healthy, age matched controls. Since GSD Ia is characterized by hypoglycaemia and previous studies have shown decreased levels of whole-body excess of reactive oxygen species as measured by specific oxidative stress markers in the patients, we hypothesized that this phenomenon could be explained by decreased formation of AGEs. However, in the present study, AGE levels, as measured non-invasively by skin $\mathrm{AF}$ and ex vivo in skin biopsies, did not differ between patients and controls. Hence, the present results do not further clarify why patients with GSD Ia avoid early atherosclerosis, as would be expected by their adverse cardiovascular risk profile, and suggest that other mechanisms may be involved in the preserved vascular health of these patients.

\section{References}

Ahmed MU, Brinkmann FE, Degenhardt TP, et al (1997) $N^{\varepsilon}$ (carboxyethyl)lysine, a product of the chemical modification of proteins by methylglyoxal, increases with age in human lens proteins. Biochem J 324(Pt 2): 565-570.

Baker L, Dahlem S, Goldfarb S, et al (1989) Hyperfiltration and renal disease in glycogen storage disease, type I. Kidney Int 35: $1345-1350$.

Berry GT, Baynes JW, Wells-Knecht KJ, et al (2005) Elements of diabetic nephropathy in a patient with GLUT 2 deficiency. Mol Genet Metab 86: 473-477.

Butler R, Morris AD, Belch JJ, et al (2000) Allopurinol normalizes endothelial dysfunction in type 2 diabetics with mild hypertension. Hypertension 35: 746-751.

Cardillo C, Kilcoyne CM, Cannon RO III, et al (1997) Xanthine oxidase inhibition with oxypurinol improves endothelial vasodilator function in hypercholesterolemic but not in hypertensive patients. Hypertension 30: 57-63.

Chen YT, Coleman RA, Scheinman JI, et al (1988) Renal disease in type I glycogen storage disease. $N$ Engl J Med 318: $7-11$.

Diamanti-Kandarakis E, Piperi C, Kalofoutis A, Creatsas G (2005) Increased levels of serum advanced glycation endproducts in women with polycystic ovary syndrome. Clin Endocrinol (Oxf) 62: 37-43.

Dyer DG, Dunn JA, Thorpe SR, et al (1993) Accumulation of Maillard reaction products in skin collagen in diabetes and aging. J Clin Invest 91: 2463-2469.

Elgawish A, Glomb M, Friedlander M, Monnier VM (1996) Involvement of hydrogen peroxide in collagen cross-linking by high glucose in vitro and in vivo. J Biol Chem 271: 1296412971.

Forbes JM, Yee LT, Thallas V, et al (2004) Advanced glycation end product interventions reduce diabetes-accelerated atherosclerosis. Diabetes 53: 1813-1823.

Fu MX, Wells-Knecht KJ, Blackledge JA, et al (1994) Glycation, glycoxidation, and cross-linking of collagen by glucose. Kinetics, mechanisms, and inhibition of late stages of the Maillard reaction. Diabetes 43: 676-683.

Hershkovitz E, Rachmel A, Ben Zaken H, Phillip M (2001) Continuous glucose monitoring in children with glycogen storage disease type I. J Inherit Metab Dis 24: 863-869.
Kiuchi K, Nejima J, Takano T, et al (2001) Increased serum concentrations of advanced glycation end products: a marker of coronary artery disease activity in type 2 diabetic patients. Heart 85: 87-91.

Lee PJ (1994) Hyperlipidaemia does not impair vascular endothelial function in glycogen storage disease type $1 \mathrm{a}$. Atherosclerosis 110: 95-100.

Levy E, Thibault LA, Roy CC, et al (1988) Circulating lipids and lipoproteins in glycogen storage disease type I with nocturnal intragastric feeding. J Lipid Res 29: 215-226.

Meerwaldt R, Oomen PHN, Links TP, et al (2004) Simple noninvasive assessment of advanced glycation endproduct accumulation. Diabetologia 47: 1324-1330.

Meerwaldt R, Hartog JW, Graaff R, et al (2005a) Skin autofluorescence, a measure of cumulative metabolic stress and advanced glycation end products, predicts mortality in hemodialysis patients. J Am Soc Nephrol 16: 3687-3693.

Meerwaldt R, Links T, Graaff R, et al (2005b) Simple noninvasive measurement of skin autofluorescence. Ann NY Acad Sci 1043: 290-298.

Meerwaldt R, Lutgers HL, Links TP, et al (2007) Skin autofluorescence is a strong predictor of cardiac mortality in diabetes. Diabetes Care 30: 107-112.

Miyata T, Fu MX, Kurokawa K, et al (1998) Autoxidation products of both carbohydrates and lipids are increased in uremic plasma: is there oxidative stress in uremia? Kidney Int 54: 1290-1295.

Monnier VM, Vishwanath V, Frank KE, et al (1986) Relation between complications of type I diabetes mellitus and collagen-linked fluorescence. N Engl J Med 314: 403-408.

Mulder DJ, Water TV, Lutgers HL, et al (2006) Skin autofluorescence, a novel marker for glycemic and oxidative stressderived advanced glycation endproducts: an overview of current clinical studies, evidence, and limitations. Diabetes Technol Ther 8: 523-535.

Mundy HR, Lee PJ (2002) Glycogenosis type I and diabetes mellitus: a common mechanism for renal dysfunction? Med Hypotheses 59: 110-114.

Nakamura Y, Horii Y, Nishino T, et al (1993) Immunohistochemical localization of advanced glycosylation end products in coronary atheroma and cardiac tissue in diabetes mellitus. Am J Pathol 143: 1649-1656.

Nieto FJ, Iribarren C, Gross MD, et al (2000) Uric acid and serum antioxidant capacity: a reaction to atherosclerosis? Atherosclerosis 148: 131-139.

Rake JP, Visser G, Labrune P, et al (2002) Glycogen storage disease type I: diagnosis, management, clinical course and outcome. Results of the European Study on Glycogen Storage Disease Type I (ESGSD I). Eur J Pediatr 161(Supplement 1): S20-S34.

Ritthaler U, Deng Y, Zhang Y, et al (1995) Expression of receptors for advanced glycation end products in peripheral occlusive vascular disease. Am J Pathol 146: 688-694.

Schleicher ED, Wagner E, Nerlich AG (1997) Increased accumulation of the glycoxidation product $N^{\varepsilon}$-(carboxymethyl)lysine in human tissues in diabetes and aging. $J$ Clin Invest 99: 457-468.

Selzer RH, Hodis HN, Kwong-Fu H, et al (1994) Evaluation of computerized edge tracking for quantifying intima-media thickness of the common carotid artery from B-mode ultrasound images. Atherosclerosis 111: 1-11.

Stegemann H, Stalder K (1967) Determination of hydroxyproline. Clin Chim Acta 18: 267-273.

Talente GM, Coleman RA, Alter C, et al (1994) Glycogen storage disease in adults. Ann Intern Med 120: 218-226. 
Terpstra WF, May JF, Smit AJ, et al (2004) Effects of amlodipine and lisinopril on intima-media thickness in previously untreated, elderly hypertensive patients (the ELVERA trial). J Hypertens 22: 1309-1316.

Thorpe SR, Baynes JW (2003) Maillard reaction products in tissue proteins: new products and new perspectives. Amino Acids 25: 275-281.

Ubels FL, Rake JP, Slaets JP, et al (2002) Is glycogen storage disease 1a associated with atherosclerosis? Eur J Pediatr 161(Supplement 1): S62-S64.

Verhave JC, Hillege HL, Burgerhof JG, et al (2005) The association between atherosclerotic risk factors and renal function in the general population. Kidney Int 67: 1967-1973.

Wells-Knecht KJ, Zyzak DV, Litchfield JE, et al (1995) Mechanism of autoxidative glycosylation: identification of glyoxal and arabinose as intermediates in the autoxidative modification of proteins by glucose. Biochemistry 34: $3702-3709$.
Wells-Knecht MC, Lyons TJ, McCance DR, et al (1997) Agedependent increase in ortho-tyrosine and methionine sulfoxide in human skin collagen is not accelerated in diabetes. Evidence against a generalized increase in oxidative stress in diabetes. J Clin Invest 100: 839-846.

Wiegman A, De Groot E, Hutten BA, et al (2004) Arterial intima-media thickness in children heterozygous for familial hypercholesterolaemia. Lancet 363: 369-370.

Wittenstein B, Klein M, Finckh B, et al (2002) Plasma antioxidants in pediatric patients with glycogen storage disease, diabetes mellitus, and hypercholesterolemia. Free Radic Biol Med 33: 103-110.

Wolff SP, Dean RT (1987) Glucose autoxidation and protein modification. The potential role of 'autoxidative glycosylation' in diabetes. Biochem J 245: 243-250.

Yudkin JS, Forrest RD, Jackson CA (1988) Microalbuminuria as predictor of vascular disease in non-diabetic subjects. Islington Diabetes Survey. Lancet 2: 530-533. 